\title{
Prolégomènes à l'analyse des coûts dans des organisations culturelles municipales
}

\author{
Stéphanie Chatelain-PonRoY ${ }^{1}$
}

\section{Résumé}

Cet article est le fruit d'une étude visant à évaluer les retombées des organisations culturelles pour leurs principaux bailleurs de fonds : les municipalités.

L'analyse du financement des organisations culturelles nous a semblé être un préalable indispensable à celle de l'analyse de leurs retombées.

MotS CLES : COUTS - FINANCEMENT - CULTURE SECTEUR PUBLIC

Correspondance :
Stéphanie Chatelain-Ponroy

Cnam-Intec

292, rue Saint Martin

75141 Paris cedex 03

Tel : 33 (0)1 53388334

ponroy@waika9.com
Abstract : Prolegomena in costs analysis of cultural organizations in the local public sector

This paper deals with a study based on how the cultural organisations value the effects they have on their main financial partners: municipalities.

The financial analysis of the cultural organisations seems to be a necessary precondition for the analysis of the consequences they have.

KEYWORDS : COSTS - FINANCING - CULTURAL ORGANISATIONS - PUBLIC SECTOR

\footnotetext{
${ }^{1}$ Maître de Conférences au Cnam-Intec, chercheur au CRC et au PESOR.

Une première version de cet article a été présentée au congrès de l'AFC de 2001. L'auteur remercie les évaluateurs anonymes de CCA pour leurs suggestions et leurs commentaires.
} 
Pour être citoyen, le ressortissant d’une collectivité locale n’en est pas moins contribuable. Il est donc bien naturel qu'il cherche à s'informer sur l'emploi que l'on fait de ses deniers, en particulier en période électorale. Une communication financière élaborée répondrait à cette préoccupation tout en permettant d'éclairer la gestion de ceux qui en ont la responsabilité. Effet secondaire peut-être, mais important, cela permettrait de “ redonner une légitimité aux élus qui souhaitent rendre compte de leur gestion ” [Lande et Ravary, 2001].

Le législateur l'a bien compris qui, par la loi ATR du 6 février 1992 et la mise en place, à partir de 1997, de l'instruction comptable M14 amendant la comptabilité publique locale, a transformé le traditionnel contrôle de conformité appuyé sur une comptabilité de caisse en une évaluation de la gestion ${ }^{\mathrm{i}}$ assise sur une comptabilité patrimoniale et d'engagement.

L’ancienne méthode visait à apprécier la qualité et la transparence des comptes par le biais de la régularité des dépenses publiques, la nouvelle devrait permettre, tout à la fois, de mesurer le niveau des services rendus et de légitimer les choix politiques et budgétaires.

En outre, ces réformes devraient permettre d'évaluer des coûts de biens ou de services, comme on le fait dans le secteur privé. C’est dans la mesure où ces coûts peuvent être connus que les gestionnaires publics ont le moyen de rendre compte de la réalisation des missions de leurs services, et de justifier l’utilisation des crédits qui leur sont alloués.

Un exemple singulièrement intéressant, quoi que difficile à cerner, est celui des activités culturelles développées par les municipalités. En effet, leur nature “ immatérielle ” ainsi que la dispersion des éléments constitutifs de leurs coûts les font échapper - provisoirement sans doute - à une évaluation strictement comptable. C’est ce qui les distingue des autres prestations pour lesquelles les réformes ont amélioré la lisibilité pour ceux qui ont la charge d’administrer les communes.

Il serait pourtant souhaitable de dégager, pour les affaires culturelles, une information aussi pertinente que pour les autres prestations à une époque où la réduction de leurs ressources 
conduit les responsables de ces organisations à s'interroger sur les voies d'une meilleure gestion de celles-ci. La conjoncture générale des finances publiques se révèle en effet très défavorable aux organisations culturelles ${ }^{\mathrm{ii}}$, les budgets de la culture servant fréquemment de variables d'ajustement pour éviter des déficits budgétaires trop importants.

Dans le même ordre d'idée, c'est devenu un topos de mettre en avant le côté élitiste de certaines organisations culturelles ${ }^{\mathrm{iii}}$, et notamment de celles qui relèvent du spectacle vivant, et de le rapporter au coût important de la culture. Doit-on dire pour autant que les subventions accordées ne servent qu’à une certaine élite sociale, qui peut ainsi fréquenter ces institutions à moindre prix, ou faut-il élargir le champ de l'analyse?

Ici se posent en réalité deux problèmes : l'évaluation des coûts et l'éventuelle nécessité de la rentabilité d’une politique culturelle.

Cet article, appuyé sur une étude de trois organisations culturelles municipales présente le premier volet de cette problématique : peut-on, et comment peut-on, inventorier les charges à incorporer à ces coûts ? Nous avons voulu ici illustrer cette préoccupations des gestionnaires, éclairer ces questions, et ouvrir des voies de recherche.

Après avoir abordé dans un premier temps une brève description des bases méthodologiques, on centrera la réflexion sur le recensement des ressources financières de ces organisations, étant entendu que les ressources des entités culturelles sont pour la plus grande part les emplois des municipalités.

\section{Les bases méthodologiques}

L’étude dont nous rendons compte ici a été réalisée entre février 2000 et janvier 2001 par trois chercheurs qui, à partir d’une problématique commune, ont développé des analyses complémentaires en contrôle de gestion, marketing, stratégie et économie, accédant ainsi à un enrichissement mutuel des analyses et à une approche plus complète de la réalité étudiée. Elle 
visait à évaluer les coûts et les retombées économiques et sociales d’organisations culturelles sur le territoire administré par leurs principaux bailleurs de fonds : les municipalités.

A la demande de la fédération d'institutions culturelles commanditaire, la recherche réalisée s'est inscrite dans le courant des études d'impact, c'est-à-dire d'études visant à mettre en évidence les effets économiques bénéfiques de la présence d'une institution sur une zone géographique considérée.

La méthodologie de ces études consiste à déterminer, inventorier, et quantifier successivement les retombées directes, indirectes et induites de l'activité observée. Ces études permettent donc d'apporter des arguments intéressants quant au poids et à l'utilité économique d'une institution puisque les flux monétaires générés localement par son activité pourront être mis en regard des subventions qui lui sont versées pour “ justifier ” économiquement l'opportunité de celles-ci, en les équilibrant en tout ou partie. Cette faculté de démonstration et de justification des investissements publics explique le succès de ces études, succès que l'on peut mesurer au nombre de travaux réalisés dans de multiples domaines ${ }^{\mathrm{iv}}$ - le secteur culturel apparaissant particulièrement bien doté à cet égard.

Les études d'impact soulèvent cependant de nombreux problèmes d'ordre méthodologique, en particulier le caractère non exhaustif des informations traitées qui amène à relativiser leur portée. Dans notre champ de recherche, il apparaissait que l'activité culturelle et l'impact de celle-ci sur son environnement présentaient une diversité et une complexité fortes nous conduisant à mesurer non seulement l'impact économique mais aussi social et qualitatif des organisations culturelles étudiées. Nous souhaitions souligner par là même le fait que pour les organisations culturelles, peut-être plus que pour n’importe quelle autre activité, le concept de performance est multipotentiel et que, par conséquent, les critères de rentabilité, de performance financière ou de productivité ne sauraient y occuper une place centrale et exclusive. Nous avons, par conséquent, élargi le champ traditionnel des études d’impact en 
nous évadant du cadre strictement économique et comptable et en intégrant des indicateurs qualitatifs ou physiques nous permettant d’appréhender l'ensemble des aspects stratégiques. Sur le plan du recueil d’informations, cette étude a mobilisé trois sources principales :

- Des questionnaires ont permis de sonder la population des organisations culturelles étudiées afin de mettre en évidence leurs principales caractéristiques et d'en déduire une typologie ${ }^{\mathrm{v}}$. Les techniques descriptives mobilisées nécessitant un échantillon de taille “ suffisante ” pour qu’il soit représentatif et pour que puissent s'appliquer les lois de la statistique -, nous avons travaillé à partir d'un questionnaire envoyé à l'ensemble des organisations membres de la fédération commanditaire de l'étude.

A l'issue de la classification hiérarchique ascendante des observations sont apparus trois groupes homogènes, chacun se distinguant des deux autres à la fois par la taille (petit / moyen / grand établissement) et par les choix stratégiques réalisés pour ses activités (“ orthodoxes ”, traduisant une fidélité aux activités traditionnelles, et “ hétérodoxes”, traduisant une certaine forme de diversification) :

- le groupe 1 rassemble des établissements caractérisés essentiellement par leur taille (petite ou moyenne) et présentant des orientations stratégiques plutôt traditionnelles ;

- le groupe 2 correspond à des maisons de taille moyenne ayant une activité très diversifiée ;

- le groupe 3 comprend des établissements de grande taille présentant une fidélité forte aux activités traditionnelles.

- Nous avons choisi de compléter et d'éclairer les résultats issus de l’analyse statistique des questionnaires par le recours à des méthodes de recueil d'informations qualitatives au travers d'études de cas. Celles-ci nous permettaient de conforter notre validation et de prétendre à une généralisation non plus statistique mais assise sur une modélisation. L'échantillon retenu n'avait, par conséquent, aucune prétention de représentativité au sens 
statistique du terme; il ne répondait qu’au souci d’éclairer l'échantillonnage par une dispersion, somme toute représentative de la réalité, en traduisant la diversité des cas possibles. La sélection des institutions a été, par conséquent, guidée par la structure même de notre problématique, et opérée dans le but de faire ressortir les contrastes entre les organisations, la marginalité apparaissant comme un facteur d'enrichissement de notre échantillon.

Dans le cadre de l'étude nous avons retenu trois établissements. Chacun d'entre eux appartenait à l’une des catégories mises en évidence par la typologie dégagée de l’analyse statistique des questionnaires.

- Enfin, une enquête a été réalisée auprès des spectateurs des trois organisations afin de mieux connaître leur public et d’évaluer les dépenses associées à leur fréquentation. 3006 questionnaires ont été ainsi analysés.

\section{Le recensement des ressources financières : la prééminence du financement communal...}

Mettre en évidence l'impact économique, sur les territoires où elles sont implantées, d’organisations culturelles suppose, au préalable, de recenser les coûts qu'elles exposent et de rapprocher ces derniers des retombées qu'elles induisent. Dans le travail que nous présentons ici, n’apparaît que le premier terme de ce rapprochement, c’est-à-dire les dépenses entraînées par l'existence même d'une institution.

L'analyse des informations collectées sur le terrain, aussi bien à l'aide des questionnaires que par l'intermédiaire des études de cas, nous permet de mettre en évidence des résultats concordants, en dépit de la variété des caractéristiques des institutions étudiées.

Il apparaît ainsi immédiatement que les coûts en question sont essentiellement composés des “ dépenses directes ” c’est-à-dire des subventions accordées par la commune, le département, la 
région et l’Etat. Celles-ci peuvent être isolées dans les comptes de l’organisation (subventions reçues) et dans celles des collectivités concernées (lignes budgétaires ou comptes annexes).

Le financement public des organisations étudiées est très déséquilibré puisque les municipalités assument plus de $60 \%$ de leurs budgets de fonctionnement :

\begin{tabular}{|c|c|c|c|c|}
\hline $\begin{array}{c}\text { Part des bailleurs de fonds publics dans les } \\
\text { budgets de fonctionnement 1999 }\end{array}$ & Etat & Région & Département & Commune \\
\hline Organisation 1 & $7 \%$ & $0 \%$ & $4 \%$ & $60 \%$ \\
\hline Organisation 2 & $7 \%$ & $7 \%$ & $0 \%$ & $70 \%$ \\
\hline Organisation 3 & $8 \%$ & $0 \%$ & $0 \%$ & $61 \%$ \\
\hline
\end{tabular}

Tableau 1 : Le financement par les bailleurs de fonds publics des trois organisations en 1999

On remarque que, pour les trois organisations étudiées, l'Etat ne participe qu'à hauteur de 7 à $8 \%$ au budget de fonctionnement, ce qui est malgré tout surprenant dans la mesure où la promotion de la culture représente l'un de ses domaines d'intervention. Les crédits affectés à la culture ne représentent, en effet, que 1\% environ du budget de la Nation, à comparer à d'autres enveloppes budgétaires comme l’éducation ou la défense.

Outre l'évidente faiblesse de l'engagement financier de l'Etat dans le domaine culturel, une critique traditionnelle de son intervention concerne la disproportion du financement entre les organisations nationales et les autres institutions. Dans le domaine de l'art lyrique, par exemple, l’Etat accorde environ 100 millions de francs par an à l'ensemble des théâtres lyriques de région alors qu'il accorde quelques 600 millions à l’Opéra National de Paris. Cette répartition apparaît peu conforme à l'équité puisque les Parisiens bénéficient de spectacles de qualité grâce à la contribution de l'ensemble des contribuables nationaux.

Le financement essentiellement communal des organisations étudiées renforce leur caractère d'équipement local et explique qu'il existe une forte appropriation de l’activité culturelle dans le discours des élus locaux. Dans les trois villes de l'enquête, les organisations font partie du patrimoine local et leur présence à proximité des mairies symbolise les liens avec le pouvoir municipal, ce qui peut constituer une garantie non négligeable pour leur pérennité. 
Ce financement, majoritairement communal, représente pour les villes un effort financier important et limite leurs capacités d'actions dans d'autres domaines. L’intervention de communes limitrophes et d'autres collectivités territoriales dans le financement des institutions culturelles permettrait de limiter cet engagement et d'équilibrer leurs efforts. Il apparaît malheureusement que les départements et les régions interviennent très peu dans le financement des organisations étudiées. Cette situation peut être considérée comme anormale, dans la mesure où les habitants de ces circonscriptions territoriales bénéficient de l'activité d’établissements dont les coûts sont majoritairement assumés par les communes dans lesquelles ils sont situés.

La répartition très inégale du financement entre les communes et les autres collectivités territoriales pourrait être expliquée par l’absence de jeux coopératifs entre ces différentes collectivités. Selon le récent rapport Chiffert, en cas de subventions obtenues par les organisations culturelles auprès d'autres collectivités territoriales, les communes apportant une contribution élevée seraient incitées à réduire conséquemment leurs subventions. Ceci peut amener les conseillers généraux et régionaux à considérer que ne seraient pas des aides réelles les subventions qu'ils pourraient leur octroyer. De même, les directeurs des institutions culturelles, accordant du crédit à cette argumentation, se dissuaderaient d'entreprendre des démarches pour obtenir des subventions départementales et régionales puisque de tels financements ne leur procureraient pas de recettes nettes. Cette façon de voir pourrait les inciter à privilégier des actions dans d’autres domaines.

\section{3. ...auquel devraient être ajoutées les ressources indirectes...}

Si les organisations étudiées reçoivent des subventions directes, apparaissant dans leurs budgets, elles bénéficient également de subventions indirectes qui correspondent principalement aux prestations qui leurs sont rendues gratuitement par d'autres services communaux. L'exemple le 
plus emblématique est la mise à disposition gratuite des bâtiments par la mairie propriétaire, les coûts de construction et de rénovation des bâtiments n'apparaissant pas dans les budgets des organisations.

Les subventions indirectes sont les dépenses effectuées au profit des institutions par les différentes collectivités mais également les tiers parties prenantes : transport des scolaires avant et après une représentation, aménagement du quartier autour de l'institution, dépenses de fonctionnement liées (sécurité, frais de réception des artistes à la mairie...), annonces gratuites des spectacles par la presse locale, lignes de bus supplémentaires les soirs de spectacle, places de parking à tarif réduit, etc.

L’évaluation de ces dépenses indirectes pose deux difficultés :

- Tout d'abord, le problème de la définition des frontières de l’organisation. En effet, ainsi que le soulignent Alain Burlaud et Claude Simon [2000], il n'existe pas de frontières naturelles clairement définies pour les institutions publiques. Ainsi, dans le cas d'une organisation culturelle, si l'on peut calculer relativement facilement le coût direct d'une représentation, encore faudrait-il y ajouter - pour en obtenir le coût complet - une quote-part des frais généraux du service culturel et de tous les services fonctionnels de la mairie, de la DRAC, du ministère de la Culture, de la chambre régionale des comptes et, pourquoi pas, des services du Premier Ministre, de l’Elysée et du parlement. La prise en compte des dépenses indirectes reflète par conséquent une opinion sur les limites de l'organisation. Pour cette étude, nous avons fait le choix de rencontrer les autorités municipales (l’organisation située sur leur territoire géographique va engendrer des coûts) ainsi que les représentants de toute autre collectivité dont le poids financier relatif est important pour l’organisation étudiée. Nous avons donc raisonné de façon marginaliste entre le coût d'accès à une information supplémentaire et l'intérêt de cette précision 
supplémentaire par rapport à l’objet de la recherche. Ce raisonnement a été appliqué à l’ensemble des dépenses indirectes (collectivités et partenaires privés).

- Par ailleurs, et si l'on suppose ces choix de définition de frontières résolus, la valorisation de ces dépenses indirectes va dépendre des informations fournies par les bailleurs de fonds (les mairies essentiellement). En effet, ces analyses n’auront de validité que si elles s’appuient précisément sur l'ensemble des dépenses liées et ne se limitent pas à l'application aux dépenses directes d'un coefficient multiplicateur, forcément entaché d'arbitraire. Or, en la matière, les informations dont nous avons disposées sont parfois parcellaires et nous ne sommes pas toujours parvenus à reconstituer les éléments manquants. En général, nos interlocuteurs ne souhaitaient pas nous les communiquer. En outre, ils peuvent considérablement varier d'une organisation à une autre, la diversité des statuts juridiques ayant pour effet d'accroître cette divergence et de rendre toute comparaison de coûts d'une institution à l'autre relativement difficile et subjective.

Affectées à des budgets communaux distincts de ceux strictement liés à l'activité culturelle ces dépenses, par nature indirectes, ne sont pas imputées au fonctionnement des organisations. Il en résulte pour la gestion des coûts de ces dernières une difficulté notoire à les quantifier, une sous-évaluation étant quasiment inévitable.

Il faut toutefois souligner que des efforts sont faits pour augmenter la transparence des comptes avec la mise en place de la norme M14 pour les comptabilités communales et le développement de budgets annexes pour leurs organisations culturelles.

Si les coûts directs sont affectés dans leurs comptes, les coûts indirects - qui sont supportés par la municipalité - peuvent quant à eux faire l'objet de retraitements et être, par un jeu d'écritures internes - les écritures d’ordre -, virés au budget annexe de l'organisation qui devrait ainsi retracer et isoler le coût réel de celle-ci supporté par le budget principal de la commune. C’est 
ainsi que, théoriquement, l'ensemble des dépenses de la commune devrait faire l'objet de retraitements et être affecté aux services concernés.

En pratique, ces retraitements concernent essentiellement les dépenses de personnel titulaire affecté à l’organisation ainsi que celles de chauffage, d'électricité, d'eau, de téléphone... Ces dépenses indirectes représentent tout de même entre $30 \%$ et $55 \%$ des budgets de fonctionnement 1999 des institutions étudiées, et constituent des éléments gérés par la ville et les services centraux municipaux sur lesquels l'équipe des personnels de l'organisation n’a aucune maîtrise... bien qu'elle en soit tenue pour responsable par l’intermédiaire de son budget.

En outre il existe des dépenses indirectes qui sont qualifiées de “ non ventilables ” c’est-à-dire qu'elles ne sont pas réparties par secteur. C’est le cas, par exemple, de certains frais d’administration liés au fonctionnement des services, ce qui signifie que la part des services généraux consacrée au fonctionnement de l’organisation ne fait pas l’objet d'une refacturation ultérieure.

On touche ici aux limites bien connues concernant les difficultés de définition d'un coût complet. En effet, de telles refacturations ne pourraient être mises en place que dans la mesure où les prestations pourraient être correctement évaluées, c’est-à-dire appuyées sur une véritable comptabilité analytique, outil encore rare dans les municipalités.

La définition des frontières du coût, c'est-à-dire de ce qui doit être affecté ou non à l'organisation, dépend à l'évidence d'une multitude de facteurs comme, par exemple, la précision du système d’information existant dans la commune ou le coût et le délai d’obtention des informations.

Ainsi, dans l'une des municipalités, l’organisation ne supporte que les seuls moyens qui lui sont directement affectés, comme les véhicules dont elle use en permanence. Ceux-ci pèseront dans son budget sous la forme d'une dotation aux amortissements, mais leur entretien ne sera pas 
enregistré dans ses comptes. Pour un véhicule temporairement mis à sa disposition, aucune charge n’est décomptée, pas même les amortissements !

Dans une autre ville, bon nombre d'éléments ne font l'objet ni de valorisations, ni d’imputations. Par exemple, les éléments qui sont réalisés par un Centre Technique Municipal n’apparaissent dans le budget de l'organisation qu'en fournitures de matières, et les charges de main d'œuvre ne sont pas valorisées puisque le personnel des ateliers n’est pas rattaché à l’organisation.

De la même manière, les frais de télécommunication des appels émanant de l'une des organisations ne lui sont pas facturés par la ville alors que ceux émanant des services de l’organisation localisés à l’extérieur de ses bâtiments sont payés directement à France Télécom par l'organisation.

Il semble en fait que les règles de rattachement soient peu claires, ne relèvent d'aucune logique et dépendent avant tout de la bonne volonté et de l’imagination des différents services municipaux, fournisseurs de prestations et de données chiffrées. Dès lors il est extrêmement difficile d'interpréter des comptes pour lesquels la règle de rattachement des charges aux budgets annexes relève d'une pratique qui peut être assez hermétique à l'esprit du non-initié.

La mauvaise lisibilité des comptes, liée à l’imbrication des éléments propres à l'organisation et de ceux de la municipalité, ne donne à l'évidence qu'une image partielle de la situation de l'institution.

En conséquence, un grand nombre de dépenses ne sera pas réaffecté au budget des organisations pour des raisons tenant plus à la réticence des acteurs locaux qu’à de véritables difficultés techniques, ainsi que le soulignait l'un de nos interlocuteurs :

“ Nous avons déjà réfléchi au niveau de la collectivité à la mise en place d'une comptabilité analytique et la refacturation interne des prestations d'un service à l'autre. Notre analyse a débouché sur le fait que cela nous obligeait à mettre en place des procédures très lourdes, dans un environnement qui n'est pas habitué à ce genre de mode de fonctionnement et qui a un manque de culture de gestion. Ce n'est pas évident de demander au serrurier des services communaux de faire une fiche d'intervention en quantifiant le temps qu'il a passé... soit on le fait par estimation au niveau central et c'est forcément imprécis - on sait 
l'imprécision des clés et le danger au niveau de l'interprétation que cela génère - soit on est obligé de descendre à un niveau très fin et l'on se retrouve devant l'écueil que nous avons cité, le risque d'incompréhension et de générer des mouvements d'humeur de la part du personnel. Alors la volonté n'a pas été de pousser plus loin ce type de réflexion ”.

Nos interlocuteurs municipaux soulignent volontiers l'impossibilité et la vanité d'un recensement complet des coûts induits par l'organisation, notamment en ce qui concerne les frais des services centraux municipaux. Notons cependant que dans certaines communes, pour plusieurs services dont celui des pompes funèbres, cette valorisation des charges des services centraux est réalisée, ce qui nous fonde à penser qu'il pourrait en être de même pour les organisations culturelles. Mais cette absence de valorisation serait due en grande partie au coût d'obtention de ces informations :

“Vous avez raison pour le coût complet mais il y a quatre-vingts services à la ville [...] et si nous faisons de la comptabilité analytique nous allons monter une usine à gaz, cela nous coûtera encore plus cher de savoir combien ça coûte. ”

L’analyse du coût, et donc du financement des organisations culturelles, devrait par conséquent intégrer l'ensemble des prestations, financées par les impôts des contribuables, dont bénéficie l’organisation étudiée.

La période pendant laquelle s'est déroulée cette étude ${ }^{\mathrm{vi}}$ n’a malheureusement pas permis de procéder à un tel recensement et la comparaison des éléments financiers d'un exercice à l'autre a été rendue délicate par le changement de système comptable auquel ont procédé les services municipaux au cours de la période étudiée (passage de la M12 à la M14).

Un suivi et une explication des variations des différents facteurs de coût ont été rendus très difficile par l'intervention commune de difficultés de natures différentes :

- Les postes d'affectation des charges peuvent changer d'une année à l'autre. En effet, les comptables publics, acquérant au fil du temps une expérience et une compétence plus grandes, analysent plus finement et plus précisément la nature des éléments à comptabiliser. Il se peut aussi que l'harmonisation comptable impose de tels choix. 
Ainsi par exemple, l’une des organisations enregistrait jusqu’à la saison 1998 / 1999 les ventes des produits dans des comptes spécifiques - 707 - qui ne correspondaient pas au formalisme comptable de la Trésorerie Municipale. Il a donc fallu réimputer ces recettes dans de nouveaux comptes - 708 - dès 1999, ce qui rend les comparaisons 1998 - 99 pour le moins délicates.

De la même manière, certains comptes regroupent indistinctement des éléments aussi divers que des locations de spectacles, des remboursements de frais de teinturier ou encore des recettes de mécénat! L’analyse de leurs variations perd de sa pertinence puisque les mouvements observés peuvent résulter aussi bien de simples reclassements que de véritables évolutions.

- Par ailleurs le compte administratif des organisations étudiées est le résultat de l'agrégation d'éléments disparates, analysés et enregistrés par des personnes distinctes. Or, en comptabilité publique il n’existe pas “ d'opérations diverses” permettant de corriger $a$ posteriori certaines imputations. Chaque correction, quelle que soit son importance, doit donc être soumise à l'approbation du conseil municipal. En conséquence, des corrections pourtant nécessaires peuvent parfois être négligées, pour éviter de lourdes procédures, au motif qu'elles touchent à des éléments qui concernent plus l'image interne de l'institution que les grands équilibres de ses comptes.

Ce que l'on vient de voir dans ce paragraphe ce sont les prestations dont bénéficient “gratuitement” les organisations et qui s’ajoutent, en termes d'intrants, aux subventions qui ont été décrites dans le paragraphe précédent.

Elles bénéficient en outre de leur “ chiffre d’affaires ”, rémunération des prestations qu’elles offrent au public. 


\section{4. ....ainsi que les recettes “ propres" de ces organisations}

Les organisations culturelles étudiées se financent également par des recettes propres de natures diverses, droits d’entrée, coproductions, vente de produits ou mécénat, les premiers constituant l'essentiel de leurs encaissements ainsi que le montre le tableau suivant :

\begin{tabular}{|l|c|c|c|}
\cline { 2 - 4 } \multicolumn{1}{c|}{} & Organisation 1 & Organisation 2 & Organisation 3 \\
\hline Part des droits d'entrée dans les budgets de fonctionnement & $23 \%$ & $10 \%$ & $24 \%$ \\
Part des droits d'entrée dans les recettes propres & $83 \%$ & $72 \%$ & $85 \%$ \\
\hline
\end{tabular}

Tableau $2:$ Le financement par les droits d'entrée des trois organisations en 1999

Ces droits d'entrée, qui mesurent la fréquentation des organisations, sont le reflet des choix stratégiques que ces dernières opèrent et qui s’expriment essentiellement par leurs choix de programmation $^{\text {vii }}$. Pour simplifier on peut opposer de façon volontairement caricaturale deux stratégies :

- La stratégie “ traditionnelle" qui se traduit par des choix de programmation homogènes basés sur des spectacles traditionnels. Selon la richesse budgétaire de l’organisation et la taille de son bassin de population, cette stratégie pourra revêtir deux modalités :

- Un choix de programmation large (nombreux spectacles et peu de représentations de chacun). L’organisation s’appuie alors sur le public des habitués et des abonnés. Ce choix nécessite des budgets importants pour créer, louer ou coproduire les nombreux spectacles de la saison. Le coût de chaque fauteuil est par conséquent élevé.

- Un choix de programmation étroit (peu de spectacles de nombreuses fois présentés). La volonté est alors d’ouvrir le programme à de nouveaux publics. C’est un choix plus difficile que le précédent car il faut attirer un nouveau public et non simplement fidéliser les habitués. Le coût de chaque fauteuil sera toutefois moins élevé que dans le modèle de programmation précédent.

- La stratégie de diversification, elle, met en œuvre des choix de programmation hétérogènes basés sur des spectacles variés. Cette stratégie permet, même en période de situation 
budgétaire tendue, de proposer au public plusieurs spectacles au cours de la saison. Il s’agit de le "faire venir à échéance régulière" et ainsi de ne pas "défidéliser une partie du public ”. Le but recherché n’est pas tant la nature et la qualité du spectacle que le nombre de représentations offertes au public, gage d'un certain équilibre financier obtenu par l'ouverture de l'organisation.

On cherchera à accueillir un public toujours plus large tout en maîtrisant le coût moyen des représentations. Des manifestations originales peuvent alors être envisagées afin d'attirer le public en dehors des sessions habituelles de fréquentation.

Par exemple, l'opéra - théâtre d'Avignon cherche à être un lieu ouvert et convivial avec les aper’opéra ou les aper'musique qui offrent des concerts de jeunes artistes - chanteurs ou instrumentistes - suivis d'une rencontre autour d'un verre offert par les vins des Côtes du Rhône. Ces manifestations connaissent un succès grandissant et témoignent du désir du public d’une fréquentation différente de l’opéra-théâtre.

Pour la municipalité ce dernier choix stratégique est beaucoup moins coûteux que le précédent. Toutefois, si l’on raisonne en terme de retombées (il s’agissait, rappelons-le, de l’objet de cette recherche) il faut souligner que celles-ci seront également bien moindres. La stratégie de diversification autorise un nombre de créations bien moins important que la stratégie traditionnelle. Or les créations induisent de longs séjours des artistes intermittents - ce qui accroît d'autant les retombées économiques directes et indirectes - et contribuent également à augmenter le pouvoir d'attraction de l'organisation, donc à développer le tourisme culturel.

Notons, enfin, que les politiques de création participent à la notoriété de l’organisation ainsi qu’à la renommée de la ville, ce qui est hors de la portée des politiques de diversification. 
Ces choix de programmation se traduisent dans l’indicateur clé de la gestion des organisations étudiées : la fréquentation. Observons, à titre d'exemple, l’influence des choix stratégiques sur le montant des droits d'entrée des organisations :

\begin{tabular}{|l|c|c|c|}
\cline { 2 - 4 } \multicolumn{1}{c|}{} & Organisation 1 & Organisation 2 & Organisation 3 \\
\hline Saison 1996 / 1997 & $9 \%$ & $10 \%$ & $16 \%$ \\
Saison 1997 / 1998 & $13 \%$ & $10 \%$ & $16 \%$ \\
Saison 1998 / 1999 & $23 \%$ & $10 \%$ & $24 \%$ \\
\hline
\end{tabular}

Tableau 3 : Part relative des droits d'entrée dans le budget de fonctionnement

Pour la première institution, on constate une augmentation très importante du poids relatif des droits d'entrée dans le budget de fonctionnement, qui traduit la diversification de l'activité et l'ouverture à un public toujours plus nombreux, mais également une diminution notable des budgets de fonctionnement alloués.

Pour la deuxième organisation, la stabilité relative des droits d'entrée dans le budget de fonctionnement est le reflet de la continuité dans la programmation, en terme de nombre de spectacles et de nombre de représentations, et de celle des budgets.

Pour la troisième, on a une progression remarquable du ratio des droits d'entrée dans le budget de fonctionnement au cours de la dernière saison, qui ne repose pas sur une augmentation de la fréquentation mais traduit un accroissement du prix de vente moyen du billet. Précisons que cette institution jouit d'un public remarquablement fidèle et relativement aisé, ce qui l'autorise à pratiquer des augmentations vigoureuses de ses tarifs.

Si les objectifs de fréquentation et la question du renouvellement du public occupent une telle place dans le discours des bailleurs de fonds, comme dans celui des responsables des organisations, c'est qu'ils comportent une double dimension :

- Une dimension stratégique, tout d'abord, car la fréquentation illustre et conditionne tout à la fois le succès ou l'insuccès de l'établissement; et même si les responsables des 
organisations réfutent l'argument de “l'audimat” comme seul indicateur de réussite, il n'en est pas moins vrai qu'ils l'utilisent volontiers pour démontrer l'importance de leur établissement et y puiser leur légitimité. Ils sont confortés dans cette position par les responsables municipaux qui affichent facilement comme principal critère de jugement “ le fait que [la salle] soit pleine toute l'année”. C’est pour eux une justification des investissements consentis.

- Une dimension opérationnelle ensuite puisque, à court terme, la fréquentation conditionne le volume des droits d'entrée, c'est-à-dire l'essentiel du “ chiffre d'affaires” de l’organisation. Son importance va dépendre de la prise en compte de deux éléments : la jauge de la salle et le prix des places.

La jauge de la salle est une donnée structurelle qui dépend du bâtiment abritant l’organisation. Il semble difficile de “ délocaliser" les productions dans des salles plus grandes pour répondre à une éventuelle demande d'un public plus important. Les organisations qui ont fait cette expérience y ont rapidement renoncé. Les décors réalisés à cette occasion, adaptés à la taille de la salle où était délocalisé le spectacle, ne pouvant être réutilisés dans l'enceinte habituelle, plus petite.

Par ailleurs, les expériences de spectacles “ hors les murs ”, permettant d’aller à la rencontre d'un nouveau public, se heurtent actuellement au problème du financement de ces spectacles. Les municipalités ne souhaitant évidemment pas financer des spectacles destinés à un public autre que leurs électeurs.

L'augmentation du prix des places fonde de nombreux espoirs peut être un peu vains puisque les responsables locaux s’opposeraient de toutes façons à une augmentation notable du prix des places au nom du principe de service public.

Pourtant, une telle décision apparaîtrait contraire à la notion même de service public, comme le faisait remarquer l'un de nos interlocuteurs : 
" Si l'on considère que nous sommes un service public il faut que le prix des places soit abordable, qu'il y ait une justice sociale. Mais l'on peut aussi avoir le raisonnement inverse en disant que plus les places sont chères, moins la différence est grande entre le prix payé et le prix réel et donc moins le contribuable supporte un art réservé le plus souvent aux classes privilégiées. C'est un discours qui est tout de même difficile à tenir, à expliquer : augmenter le prix des places de deux cents francs pour être plus populaire... ce n'est pas simple à expliquer, mais ce n'est pas faux. ”

Cet avis est partagé par l'ensemble de nos interlocuteurs - au sein des organisations comme dans les services bailleurs de fonds - qui jugent une augmentation du prix des places antinomique avec la mission de service public des organisations culturelles :

"Nous sommes un service public. [...] La Culture c'est un choix. Un service culturel est par nature déficitaire. [...] Nous avons la possibilité de rendre la Culture ouverte à tous, populaire. C'est la mission de l'Etat et des collectivités territoriales."

Les organisations culturelles françaises, ou du moins leurs autorités de financement, utilisent leur politique tarifaire comme symbole et outil de leur politique d'ouverture à un large public. La politique culturelle des municipalités étudiées comporte en effet toujours un volet visant à permettre une répartition plus homogène de l'offre culturelle municipale entre tous les habitants.

Les organisations culturelles, qui s’adressent traditionnellement à un public appartenant à une certaine élite sociale, sont des emblèmes de cette politique et se doivent donc d'être ouvertes au plus grand nombre. C’est ainsi que les tarifs généraux s’échelonnent entre trente francs et plusieurs centaines de francs selon les institutions, le genre du spectacle, la notoriété du titre proposé et la place occupée.

De la même manière, les organisations offrent des réductions souvent très importantes sur les abonnements alors que certains établissements culturels étrangers, comme l’opéra de Genève par exemple, choisissent, au contraire, de fixer le tarif des abonnements à un niveau plus élevé que les prix des billets individuels.

De surcroît, l'augmentation du prix moyen des droits d’entrée est également combattue par ceux de nos interlocuteurs qui soulignent volontiers le fait, non démontré, qu’une 
augmentation du prix des places pourrait amener une évaporation importante des spectateurs habitués.

Cette double limite, des quantités de places offertes et des prix pratiqués, explique le déficit chronique dont souffre la plupart des organisations vouées au spectacle vivant :

" Chaque fois que nous levons le rideau, hélas, nous sommes déficitaires. Oui, bien sûr on peut équilibrer: aux Chorégies [d'Orange] quand ils jouent devant huit mille personnes qui paient chacune huit cents francs, la jauge de la salle de plusieurs millions de francs permet d'équilibrer un coût de plateau ".

Ce recensement des ressources financières des organisations culturelles nous a permis d’approcher au plus près les coûts engendrés par chacune pour ses différents bailleurs de fonds. Cependant, et ainsi que le notait Madame la ministre de la culture et de la communication lors de la séance des questions au gouvernement à l’Assemblée Nationale :

“ Les éléments chiffrés permettant de suivre les financements et les activités des [organisations culturelles] en région [...] sont délicates à établir. Les données recueillies divergent parfois selon les sources de façon importante et ne permettent pas toujours d'effectuer des comparaisons pertinentes "wiii.

Pas plus que le ministère de la Culture nous n’avons pu totalement contourner cette difficulté dont le traitement nécessiterait un travail extrêmement approfondi auprès de chaque organisation et de ses bailleurs de fonds et un accès sans restriction aucune aux différents éléments chiffrés.

La première de ces difficultés tient au fait que les budgets publics sont construits selon un rythme d'annualité civile, alors que les organisations culturelles utilisent en principe la saison comme référence de leur travail artistique.

Par ailleurs, tous les budgets étudiés n’incorporent pas les mêmes éléments. C’est ainsi, par exemple, que le coût de l’orchestre attaché à un opéra peut être globalisé dans les comptes de ce dernier ou faire l'objet d’une valorisation spécifique. Dans ces conditions, le service rendu par l’orchestre peut alors difficilement être évalué et comptabilisé. 
Enfin, les dépenses induites par le fonctionnement de l'organisation au sein de la collectivité à laquelle elle est attachée sont valorisées selon des procédures non homogènes d'une municipalité à l'autre, mais également d'une dépense à l'autre, pour une même municipalité.

Ces difficultés n’apparaissent pas seulement dans notre champ d’étude. Pour lui, comme pour d'autres activités publiques, c'est aujourd'hui une pierre d'achoppement particulièrement aiguë. En effet, et ainsi que nous le soulignions en introduction, les réformes qui touchent actuellement les systèmes d'information publics font évoluer ceux-ci d'un contrôle formel centré sur les procédures et la conformité des réalisations aux décisions budgétaires à un contrôle de la performance et des moyens alloués.

Dans le même ordre d’idée, les contrôles exercés par les Chambres Régionales des Comptes ne portent plus sur la seule régularité des décisions des collectivités territoriales, mais sur l’efficacité des dépenses engagées en s'efforçant d'en faire des bilans coût-efficacité.

De même encore, se développent des pratiques d'évaluation des actions publiques cherchant à approcher les notions d'impact et de satisfaction des différents segments de la population.

La simplification des règles budgétaires, en offrant une plus grande liberté d'action aux gestionnaires publics, a renforcé leur pouvoir mais aussi leurs responsabilités et pourrait les conduire à rendre compte a posteriori de la réalisation de leurs objectifs et de leurs performances en terme d'efficacité et d'efficience.

Ceci suppose évidemment de poursuivre le travail engagé avec la réforme de la comptabilité publique par un développement de nouveaux outils de mesure et de contrôle pour lesquels le recensement exhaustif et rigoureux des ressources financières, et donc des coûts, constitue un préalable indispensable.

Ceci est de plus en plus vrai alors que se multiplient les structures intercommunales et qu'il n’existe pas de système d’information permettant de consolider, pour une même activité, 
l'ensemble des données financières des structures locales. Un tel système d'information constituerait pourtant la base de données à partir de laquelle les élus pourraient rendre compte de leur politique et en être responsables vis-à-vis des électeurs.

L’étude à caractère exploratoire que nous avons présentée ici montre qu’on ne dispose ni de l’outil théorique - ne serait-ce que la structure des coûts - ni des moyens pratiques de réaliser totalement cette consolidation. Si des embryons de système d'information existent, ils mériteraient d’être approfondis et développés.

\footnotetext{
${ }^{\mathrm{i}}$ Meilleure lisibilité des comptes et plus grande proximité avec la réalité économique.

${ }^{\text {ii }}$ V.A. (2000), Les dépenses culturelles des collectivités locales en 1996, Développement culturel, Ministère de la Culture.

iii En particulier de celles qui appartiennent à la culture dit " légitime ”.

${ }^{\text {iv }}$ Cf., par exemple, dans le domaine de la culture, les études réalisées par Abbé-Décarroux (1989) sur le rôle économique du Grand Théâtre de Genève ou par le Forum Régional d'Etudes Spécifiques (1998) sur l'impact économique des activités du secteur de la culture de Montréal.

${ }^{v}$ Analyse en composantes principales pour identifier la structure des variables puis classification hiérarchique ascendante permettant de construire une typologie des organisations étudiées.

${ }^{\text {vi }}$ Les comptes étudiés étaient ceux de 1997, 1998 et 1999.

vii On peut penser que la stratégie des organisations culturelles relève d'une démarche plus volontariste que réactive puisqu'elle est le reflet du projet du directeur de l'organisation auquel elle est adossée. Il faut toutefois souligner que le contexte budgétaire et les variations des budgets alloués à ces organisations vont influer directement sur les choix stratégiques de ces dernières.

viii Journal officiel du 31 mai 1999.
}

\section{Bibliographie}

ABBE-DECARROUX F., Le rôle économique du Grand Théâtre de Genève, Etude pour le département municipal des affaires culturelles de la ville de Genève, 1989.

BONNAFOUS-BOUCHER M., CHATELAIN-PONROY S., EVRARD Y., MAZALLON F. et alii., Etude des coûts et des retombées directes, indirectes et qualitatives des théâtres lyriques, rapport d'étude commandé par la chambre professionnelle des directeurs d'opéra au cabinet mbbc, 2000.

BURLAUD A., SIMON C., Comptabilité de gestion. Coûts / Contrôle, Vuibert, 2000.

CHATELAIN S., Le contrôle de gestion dans les musées, Paris, Economica, 1998.

CHIAPELLO E., Les modes de contrôle des organisations artistiques, Doctorat en Sciences de Gestion, 1994, Université Paris IX Dauphine.

CHIFFERT A., LECAT R., RELIQUET P., La rénovation des instruments juridiques des services publics culturels locaux, Ministère de la Culture et de la Communication, nº 1999 / 18, février 1999.

CONSEIL ECONOMIQUE ET SOCIAL, “ L'évaluation des politiques publiques ”, Journal Officiel, 1990.

DRESSAYRE P., GARBOWNIK N., "Le manager imaginaire ou les illusions du management public de la culture ”, Communication au colloque international " Politiques et Management Public ”, 1992. 
DUPUIS X., La prise en compte de la dimension culturelle du développement : un bilan méthodologique? , UNESCO, division du développement culturel, 1988.

DUPUIS X., L'économie méconnue de l'Opéra, Laboratoire d'économie sociale, Intergroupe de recherche en économie de la culture, Université de Paris I Panthéon-Sorbonne, 1996.

EVRARD Y. (ed.), Le management des entreprises artistiques et culturelles, Paris, Economica, 1993.

FORUM REGIONAL D'ETUDES SPECIFIQUES (FRES), Impact économique des activités du secteur de la culture des cinq régions du Montréal métropolitain et de la région de l'île de Montréal, Gouvernement du Québec, décembre 1998.

FRANÇOIS-NOYER V., "Evaluation des politiques publiques locales et contrôle de gestion : nature d'une relation ”, Les cahiers de la recherche du CLAREE, IAE Lille, 1996.

FUMAROLI M., L'Etat culturel, Editions de Fallois, 1991.

HARVEY J., Le sport au Canada : poids, impact économique et rôle du gouvernement, Ecole des sciences de l'activité physique, Université d'Ottawa, janvier 1998.

HERSCOVICI A., la demande suscitée dans le domaine culturel, CNFPT, mars 1989.

KIEFFER R., MERLIERE Y., “L'expérience de l'assistance publique - hôpitaux de Paris dans la mise au point d'indicateurs d'évaluation économique des activités hospitalières ”, Journées de recherche des IAE, Nantes, 1998.

LANDE E., RAVARY L., Comptabilité publique locale, Cnam-Intec, 2002.

MEYSSONIER F., Compte-rendu d'une enquête sur les systèmes d'information et de gestion des municipalités, Communication au douzième congrès de l'Association Française de Comptabilité, 23, 24 et 25 mai 1991.

RAVARY L., Rubrique mensuelle “Des nouvelles de la comptabilité publique”, Revue Française de Comptabilité.

REVERCHON A., “ Comment gérer la culture ? ”, Le Monde, 26 et 27 février 1989.

ROUX (de) E., “ Avignon mal à l’aise en “ capitale culturelle de l’Europe ” ”, Le Monde, 2000.

SERROU B., L'opéra en région, rapport commandé par Alain Juppé et Philippe Douste-Blazy, 1998.

TRAUTMANN C., Les mesures nouvelles en faveur de la démocratisation de la culture, Communication en Conseil des ministres, 23 juin 1999.

V.A., “ Administrer la culture ? ”, Revue Française d'Administration Publique, № 65, janvier - mars 1993.

V.A., “ Culture et société ”, Les cahiers français, la documentation française, mars - avril 1993.

V.A., “ Guggenheim, Bilbao ”, Connaissance des arts, HS nº 134, 1999.

V.A., “ Le management, un défi pour les organisations non marchandes ”, Gestion 2000, 1 - 1992.

V.A., "Les dépenses culturelles des collectivités locales en 1996 ”, Développement culturel, Ministère de la Culture, 2000.

V.A., Quatrième conférence internationale sur l'économie de la culture, Avignon, 1986, La Documentation Française (4 volumes).

V.A., “Questions écrites, réponses des Ministres, Culture et Communication ”, Journal Officiel, lundi 31 mai 1999.

V.A., “ Recherche : Culture et Communication ”, 64 ème congrès de l’ACFAS, Université Mc Gill, Montréal, 15 et 16 mai 1996.

VERAN L., CANAS A., “Quelques clés pour gérer les activités culturelles, Quand le chercheur en gestion s'interroge sur le secteur culturel ”, Revue Française de Gestion, mars - avril - mai 1987. 